SLAC-TN-12-029

\title{
Optical Design of a Broadband Infrared Spectrometer for Bunch Length Measurement at the Linac Coherent Light Source
}

\author{
Kiel Williams \\ Office of Science, Science Undergraduate Laboratory Internship (SULI) Program \\ Guilford College, Greensboro, North Carolina \\ SLAC National Accelerator Laboratory \\ Menlo Park, California
}

20 August, 2011

Prepared in partial fulfillment of the requirements of the Office of Science, U.S.

Department of Energy's SULI Program under the direction of Dr. Josef Frisch at the Linac Coherent Light Source (LCLS).

Participant:

Signature

Research Advisor:

Signature 
Table of Contents

$\begin{array}{ll}\text { Introduction } & 4\end{array}$

$\begin{array}{lr}\text { Materials and Methods } & 6\end{array}$

$\begin{array}{lr}\text { Results } & 9\end{array}$

$\begin{array}{ll}\text { Discussion } & 11\end{array}$

$\begin{array}{ll}\text { Acknowledgements } & 12\end{array}$

$\begin{array}{ll}\text { References } & 13\end{array}$

Tables and Figures $\quad 14$ 


\begin{abstract}
The electron pulses generated by the Linac Coherent Light Source at the SLAC National Accelerator Laboratory occur on the order of tens of femtoseconds and cannot be directly measured by conventional means. The length of the pulses can instead be reconstructed by measuring the spectrum of optical transition radiation emitted by the electrons as they move toward a conducting foil. Because the emitted radiation occurs in the mid-infrared from 0.6 to 30 microns a novel optical layout is required. Using a helium-neon laser with wavelength $633 \mathrm{~nm}$, a series of gold-coated off-axis parabolic mirrors were positioned to direct a beam through a zinc selenide prism and to a focus at a CCD camera for imaging. Constructing this layout revealed a number of novel techniques for reducing the aberrations introduced into the system by the offaxis parabolic mirrors. The beam had a recorded radius of less than a millimeter at its final focus on the CCD imager. This preliminary setup serves as a model for the spectrometer that will ultimately measure the LCLS electron pulse duration.
\end{abstract}




\section{INTRODUCTION}

The Linac Coherent Light Source (LCLS) at the SLAC National Accelerator Laboratory is a free-electron laser (FEL) capable of producing some of the shortest $\mathrm{x}$-ray pulses ever observed. The LCLS produces electron pulses less than ten femtoseconds in duration. These pulses then emit soft and hard x-rays as magnetic fields force the electron bunches to oscillate back and forth [1].

Due to the short duration of the LCLS electron pulses, a precise measurement of their length has proven difficult to obtain [2]. Improved information on the pulse length would enable researchers to better understand the time scales over which the processes they observe are happening. The dissolution of chemical bonds and the folding of different types of protein are known to happen over scales similar to the length of LCLS electron pulses, and more knowledge of the pulse length could be translated into more a knowledge of these fundamental reactions.

An indirect measurement of the electron pulse length can be obtained by observing the optical transition radiation $(\mathrm{OTR})^{1}$ given off by the electron pulses as they strike a conducting material [3]. The OTR can then be directed through an optical layout and toward a thermal detector that records the constituent spectrum.

Due to the broadband nature of the spectrum of OTR emitted by the electrons, the internal configuration of this spectrometer differs significantly from that of most modern spectrometers. Most modern spectrometers focus on too narrow a spectral band to appropriately analyze the electrons' full OTR band. Conventional glass optical materials also fail to transmit

\footnotetext{
${ }^{1}$ The induced mirror charge on the conductor and the approaching electron together behave like a collapsing electric dipole as the electron approaches, with the emitted OTR falling in a wide spectral band primarily within the infrared [4].
} 
over wavelengths comparable to that of the electron bunch OTR (0.5-30 microns). Additionally, typical diffraction gratings fail to examine a wide enough spectral band to sufficiently analyze the OTR. Triangular prisms can decompose light across a wide range of frequencies into its constituent wavelengths, permitting such prisms to act as appropriate substitutes. Figure 1 illustrates the relevant geometry of this type of prism. The total deviation angle of a light ray $\gamma$ through such prisms is given by

$$
n \sin \frac{\gamma+\varepsilon}{2}=n_{0} \sin \frac{\varepsilon}{2}
$$

where $n$ and $n_{0}$ are the indices of refraction for the outside medium and the inside of the prism respectively, and $\varepsilon$ is the apex angle of the prism (aberrational effects through the prism are minimized for this configuration). Furthermore, when the prism is in this aberration ${ }^{2}$ minimizing configuration it can be shown that the relation

$$
\cos \alpha=n_{1} \sin \frac{\gamma+\varepsilon}{2}
$$

where $\alpha$ is angle formed by the incident light ray and the surface of the prism, also holds.

Expanding beam optics can be used as a means of bringing a beam parallel to a chosen axis. One concave and one convex lens can accomplish this in a simple case. A diagram of this setup is illustrated in Figure 2. The magnitude of magnification $M$ obtained by such a setup is given by the relation

$$
M=\frac{f_{2}}{f_{1}},
$$

where $f_{2}$ is the focal length of the convex lens, and $f_{1}$ is the focal length of the concave lens.

\footnotetext{
${ }^{2}$ In this paper an aberration is taken to be any optical effect that degrades the circularly-symmetric Gaussian profile of the beam.
} 
For a Gaussian mode $\mathrm{TEM}_{00}$ beam, analysis of the analytic expression for the beam profile admits numerical formulae for the half-width and $1 / \mathrm{e}^{2}$ width of the beam in terms of one another. Frequently the half-width can be measured with greater ease and converted to the $1 / \mathrm{e}^{2}$ width based on the formula

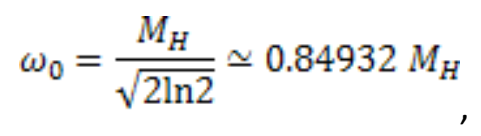

where $\omega_{0}$ is the $1 / \mathrm{e}^{2}$ radius of the beam and $M_{H}$ is the full-width of the beam at half of its maximum power.

\section{MATERIALS AND METHODS}

Preliminary construction of the optical layout utilized a 632.8 nanometer wavelength helium-neon mode $\mathrm{TEM}_{00}$ laser of minimum peak power $0.8 \mathrm{~mW}$ and a $1 / \mathrm{e}^{2}$ diameter of 0.48 millimeters. A series of reflecting mirrors and lenses were inserted to expand and direct the beam. A plano-concave and plano-convex lens were placed the difference of their focal lengths apart to enable their operation as a Galilean beam expander. The projected final beam diameter was calculated according to Equation 3, and the final diameter of the beam was measured by ruler as it emerged from the convex lens onto a portable screen. Gold-coated plane mirrors then directed the beam towards a collection of gold-coated off-axis parabolic (OAP) mirrors, with irises aligning the beam along each portion of its path. The first two OAP mirrors were separated by the sum of their focal lengths, allowing the beam to further expand as it passed from the first to the second. A zinc selenide ( $\mathrm{ZnSe}$ ) triangular prism of apex angle 10 degrees was placed approximately $50 \mathrm{~mm}$ in front of the second OAP mirror. A prism made of thallium- 
bromoiodide will be used in the final spectrometeter, but due to its toxic properties and great fragility $\mathrm{ZnSe}$ was used for this preliminary setup.

A third gold-coated OAP mirror was placed $71 \mathrm{~mm}$ in front of the prism. Equation 1 dictated based on the prism apex angle that aberrational effects would be minimized for a total deviation angle of approximately 16.9 degrees. The third OAP mirror was then positioned 21 $\mathrm{mm}$ to the side of the prism in such a way that it would intercept the incoming beam at this optimal angle. The orientation of the ZnSe prism was then adjusted until the beam reflected off of the positioned third OAP mirror. Equation 2 dictates a backscatter angle $\alpha$ of 76.9 degrees for optimal aberration reduction. Fine adjustments to the tilt of the $\mathrm{ZnSe}$ prism were performed until a backscatter angle closely echoing this theoretical optimum was measured. The backscatter angle was measured by blocking the backscattered beam with a screen, measuring the distance from the prism to the screen, and comparing this distance with the length between the second OAP mirror and the backscatter-blocking screen. The backscatter angle could then be extracted from the situational geometry. The third OAP was also adjusted to reduce aberrational effects as it intercepted and reflected the beam.

The focal point of the third OAP mirror was located as the laser reflected from it using a screen and an adjustable slit capable of accurately recording widths on the order of the hundreds of microns was positioned there. A power meter was used to detect the drop-off in power of the beam as the slit was closed, recording the width at which the power dropped to half its initial peak. This width was converted to the $1 / \mathrm{e}^{2}$ diameter of the beam using Equation 4 . The slit was then repositioned to the shared focal point between the first and second OAP mirrors. Two adjustable slides were attached to the base of the slit and it was moved to the focal point of the first OAP mirror by sliding it alternatively towards and away from the OAP mirror. The point at 
which sliding the slit perpendicular to the beam reversed the direction the image moved on a mounted screen was scanned for as a means of precisely locating the focal point of the first OAP mirror. Each time the beam image switched the direction it moved relative to the horizontal slide of the stage it was revealed that the focal point had been passed. This process could then be iterated to bring the slit closer to the focal point. Following these first-order positioning corrections the slit size was adjusted and it was moved in a way that maximized the amount of passing light. The horizontal and vertical knobs on the first OAP mirror before the slit were also adjusted as a means of eliminating beam aberrations introduced by this mirror into the optical system. A measurement of the beam waist using the same power-based method as for the prior focal point after the third OAP mirror was taken and compared with simulated data [6]. A measurement of the beam waist at its origin from the laser was also performed in this manner.

A CCD camera fitted with a $30 \mathrm{~dB}$ circular attenuator was then placed at the final focal point to profile the beam at the end of the optical layout and connected to a nearby computer terminal. Placing the CCD camera so that its surface formed an angle of approximately 44 degrees with the incoming beam allowed for further beam aberration minimization based on the results of previous optical simulations. Figure 3 illustrates this configuration of optical components. Table 1 records the focal length of each lens and OAP mirror.

Precision alignments of the OAP mirrors following their initial placement were performed by first rotating them 180 degrees and observing that the beam profile fell on the center bolt on the rear of each mirror. It was verified that each OAP was at an appropriate height by doing this in turn to each of the three OAP mirrors in the system. The horizontal and vertical fine adjustment knobs on the OAP mirrors then enabled the beam to be gradually directed through the last half of the system and to the center of the CCD camera. 
Positioning a small screen immediately after each of the three OAP mirrors then allowed an assessment of the extent to which they introduced damaging aberrations into the beam profile. The methods used to reduce the observed aberrations in the OAP mirrors included small rotations along the optical axis of the mirrors and minor height adjustments to better center the incoming beam, and turning the OAP mirrors while they rested in their holders to alter the angle at which they deflected the incoming beam. Adjustment of this tilt enabled the beam to maintain its six-inch height in addition to the correction of aberrational effects. Adjusting the micrometer on the slit after the first OAP mirror and observing the laser profile on a screen also guided aberrational corrections. Figure 4 illustrates the final layout of the aligned optical components.

A set of images of the beam was collected immediately after concave lens at the beginning of the optical layout with the CCD camera to ensure its linearity. Images of the beam at different widths as it grew larger through the beam expander were collected by shifting the position of the camera. These images were then loaded into Matlab ${ }^{\mathrm{TM}}$ and the intensities across a center cut of the beam were summed together. By comparing this summation for the beam at different widths, the degree to which nonlinear digital effects applied by the CCD camera impacted images of the beam and its accompanying intensity at points throughout the optical system was determined.

\section{RESULTS}

At its origin the beam $1 / \mathrm{e}^{2}$ diameter was measured as $.28 \pm .02 \mathrm{~mm}$ using the slit micrometer. Following the passage of the beam through the Galilean expander it attained a diameter of $6 \pm 1 \mathrm{~mm}$ based on an approximate ruler measurement (the size of the beam at this point in the system made a micrometer measurement impractical). This measurement is in line 
with that predicted by Equation 3. Additionally, CCD camera images taken at the conclusion of the optical layout reveal the beam to have an approximately Gaussian structure when the slit was left fully open. Summing the Matlab ${ }^{\mathrm{TM}}$ intensity indices of different cross-sections of the beam taken with the CCD camera revealed an image-to-image variation of less than five percent. Figure 5 shows an image of the beam at this focal point of the third OAP mirror under $50 \mathrm{~dB}$ of total attenuation (the additional $20 \mathrm{~dB}$ of attenuation resulted from a second attenuator placed immediately in front of the CCD camera).

In the final optical configuration a total deviation angle of $15 \pm 1$ degrees through the prism was measured. Measure of the backscatter angle after adjustment revealed a backscatter angle of 73 degrees to within an error of less than five degrees. This falls within five degrees of the theoretical optimum angle given by Equation 2 to reduce aberrational effects. Small variations in this angle were not observed to have a major impact on the reduction of aberrational effects.

A beam profile diameter of $173 \pm 5$ microns was recorded at the focal point of the first OAP mirror using the slit micrometer. Using the slit to measure the beam profile at its focus after the third OAP mirror yielded a measurement of approximately $237 \pm 5$ microns for the $1 / \mathrm{e}^{2}$ beam diameter at this point in the optical layout. Performing fine adjustments to the orientation of each OAP mirror and positioning the slit carefully between the first and second OAP mirror eliminated many aberrations throughout the system. Initially, the beam appeared to diminish from the vertical direction as it passed through the horizontal slit following the first OAP mirror. By empirically adjusting the horizontal and vertical tilt of the first OAP mirror this aberration has been mitigated. The beam continues to focus in two different planes as it approaches the focal point of the third OAP mirror. 


\section{DISCUSSION}

Significant reductions in the severity of optical aberrations throughout the final system proved attainable through hand adjustment of the constituent optics. Following the initial placement of the optical equipment the beam focused horizontally and vertically in different planes in the space following the third OAP mirror. Rotating the third OAP mirror in a way such that the beam fell precisely at its center helped to reduce the problem somewhat. Rotating the tilt of the angle at which the second OAP mirror intercepted the incoming beam proved helpful as well. Errors in the angle of the tilt of OAP mirrors about their optical axes of less than one degree can greatly distort the final image by bending it into an arc-like pattern. Adjustment of this tilt itself proved one of the more effective means of determining the correct tilt angle for the OAP mirrors due to the observable aberrations introduced by even small initial errors.

Simulations predict a beam diameter of 65 microns at the focal point of the first OAP mirror, and a required tilt of 0.4 degrees of the first OAP mirror to attain the measured $1 / \mathrm{e}^{2}$ diameter of $173 \pm 5$ microns. Similar simulations project a spot size of 38 microns at the focal point of the third OAP mirror, with a required tilt of approximately 1 degree to attain the measured $1 / \mathrm{e}^{2}$ diameter of $237 \pm 5$ microns. This is less consistent with expected human alignment errors, and suggests deeper aberrational effects occurring in the second and third OAP mirror, and potentially within the prism as well.

One aberration of particular note is that which occurs immediately after the closure of the slit in which the beam would appear horizontally oriented despite the vertical orientation of the slit. Noting that this aberration coupled the horizontal and vertical propagation of the beam, the horizontal and vertical tilt of the first OAP mirror were gradually changed until the beam was oriented vertically as it exited the slit. Because altering the vertical and horizontal mirror tilt 
iteratively proved effective in eliminating the problem, it is suggested that the way the OAP mirror couples the propagation of the beam in the horizontal and vertical direction causes the beam to twist in some manner as it passes through the slit. Noting patterns like these will become a progressively more important means of correcting aberrations in later versions of the spectrometer as lasers in the near- and mid-infrared are incorporated into the design.

Future modifications to this preliminary layout will incorporate a pyroelectric thermal line detector that will ultimately be used to capture the spectrum of the LCLS beam [7]. The orientation of the beam as it reflects from the third OAP mirror will require a greater level of attention due to the sensitivity of the detector. In conjunction with this, additional lasers emitting in the near- and mid-IR spectrum will be added to enable calibration of the sensor and test how the optical layout transmits signals through portions of the IR spectrum.

\section{ACKNOWLEDGEMENTS}

This work received support from the SLAC National Accelerator Laboratory and Department of Energy, Office of Science. I would like to thank my mentor Josef Frisch for the assistance he provided throughout this project. I also wish to thank Alan Fisher, Mark Petree, George Burgueno, and my research partners Julie Cass and Gilles Dongmo-Momo for their invaluable assistance. 


\section{REFERENCES}

[1] Z. Huang, A. Baker, C. Behrens, M. Boyes, J. Craft, F.-J. Decker, Y. Ding, P. Emma, J. Frisch, R. Iverson, J. Lipari, H. Loos, D. Walz, "Measurement of Femtosecond LCLS Bunches Using the SLAC A-Line Spectrometer." Paper presented at the 2011 Particle Accelerator Conference, New York, New York, March 28-April 1, 2011.

[2] M. Zelazny, S. Allison, S. Chevtsov, P. Emma, K.D. Kotturi, H. Loos, S. Peng, D. Rogind, and T. Straumann, "Electron Bunch Length Measurement For LCLS at SLAC." Paper presented at the 2007 International Conference on Accelerator and Large Experimental Physics Control Systems, Knoxville, Tennessee, October 15-19, 2007.

[3] J. Frisch, R. Akre, F.-J. Decker, Y. Ding, D. Dowell, P. Emma, S. Gilevich, G. Hays, P. Hering, Z. Huang, R. Iverson, R. Johnson, C. Limborg-Deprey, H. Loos, E. Medvedko, A. Miahnahri, H.-D. Nuhn, D. Ratner, S. Smith, J. Turner, J. Welch, W. White, J. Wu, "Beam Measurements at LCLS." Paper presented at the Beam Instrumentation Workshop, Lake Tahoe, California, May 4-8, 2008.

[4] C. Behrens, A. Fisher, J. Frisch, S. Gilevich, H. Loos, J. Loos, "Design of a Single-Shot Prism Spectrometer in the Near- and Mid-Infrared Wavelength Range for Ultra-Short Bunch Length Diagnostics." Paper presented at the $10^{\text {th }}$ European Workshop on Beam Diagnostics and Instrumentation for Particle Accelerators, Hamburg, Germany, May 16-18, 2011.

[5] C. Behrens, "Design of an Infrared Prism Spectrometer for Ultra-Short Bunch Length Diagnostics." Presentation at a meeting of the SLAC National Accelerator Laboratory Accelerator Physics and Engineering Department, Menlo Park, California, November 30, 2010.

[6] J. Cass, "Simulations and Analysis of an Infrared Prism Spectrometer for Ultra-Short Bunch Length Diagnostics at the Linac Coherent Light Source.” Produced for the Department of Energy Student Undergraduate Laboratory Internship program at the SLAC National Accelerator Laboratory, Menlo Park, California, 2011.

[7] G. Dongmo-Momo, "Infrared Spectroscope for Electron Bunch-length Measurement: Heat Sensor Parameters Analysis." Produced for the Department of Energy Student Undergraduate Laboratory Internship program at the SLAC National Accelerator Laboratory, Menlo Park, California, 2011. 


\section{TABLES AND FIGURES}

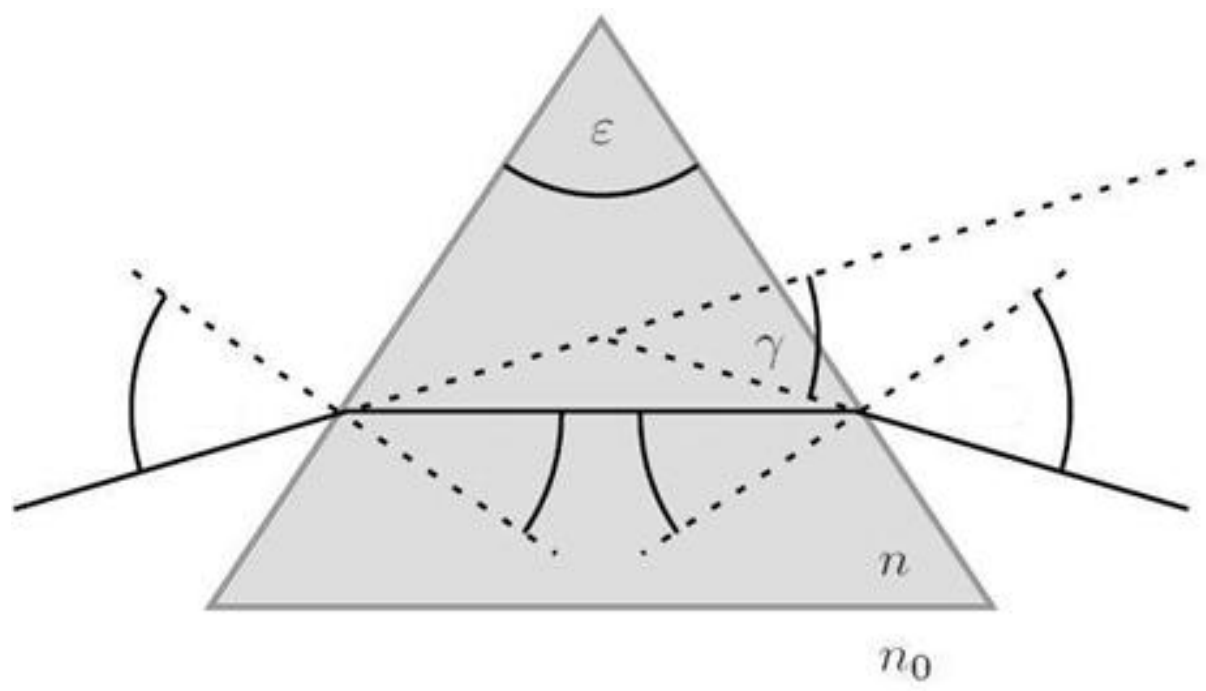

Figure 1: Illustration of a prism in an aberration-minimizing symmetric configuration. Note that all incoming and outgoing angles are equal to their symmetric partners. Courtesy C. Behrens. 


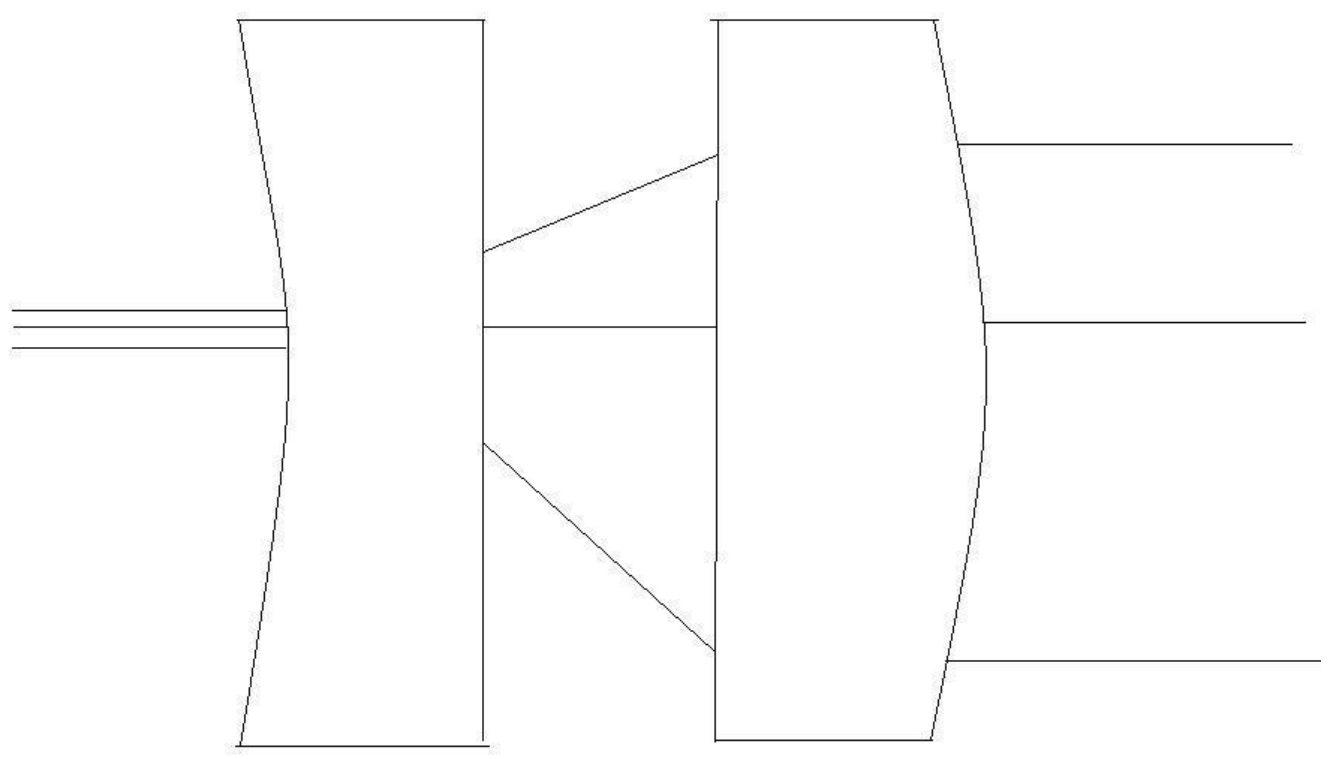

Figure 2: Illustration of the lens configuration that expanded the beam in this optical layout. Note that the lenses are separated by the difference of their focal lengths. 


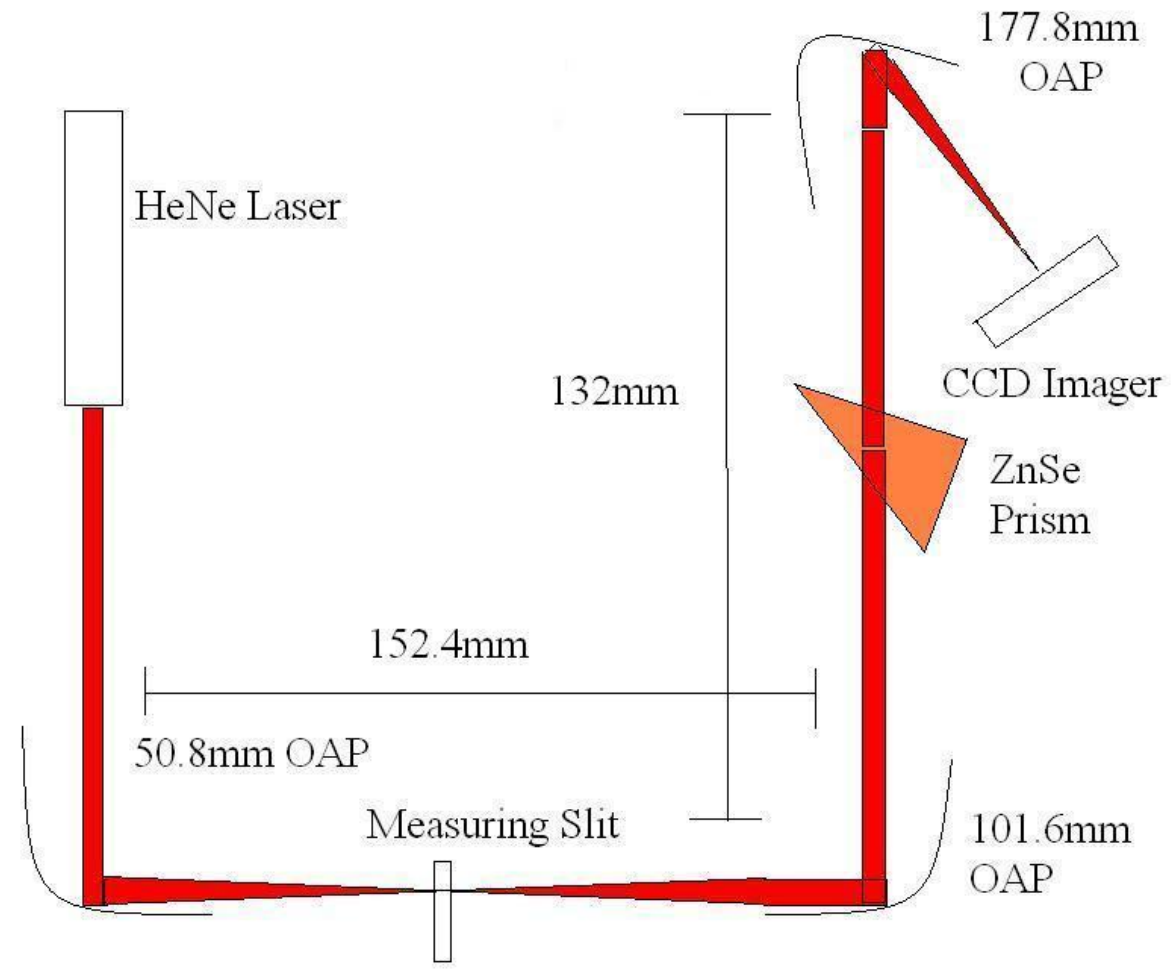

Figure 3: Illustration of the optical layout directing the helium-neon beam to a final focus. Note that this is one of several placements used for the measuring slit. The lens were placed before the first OAP mirror and are not shown. 


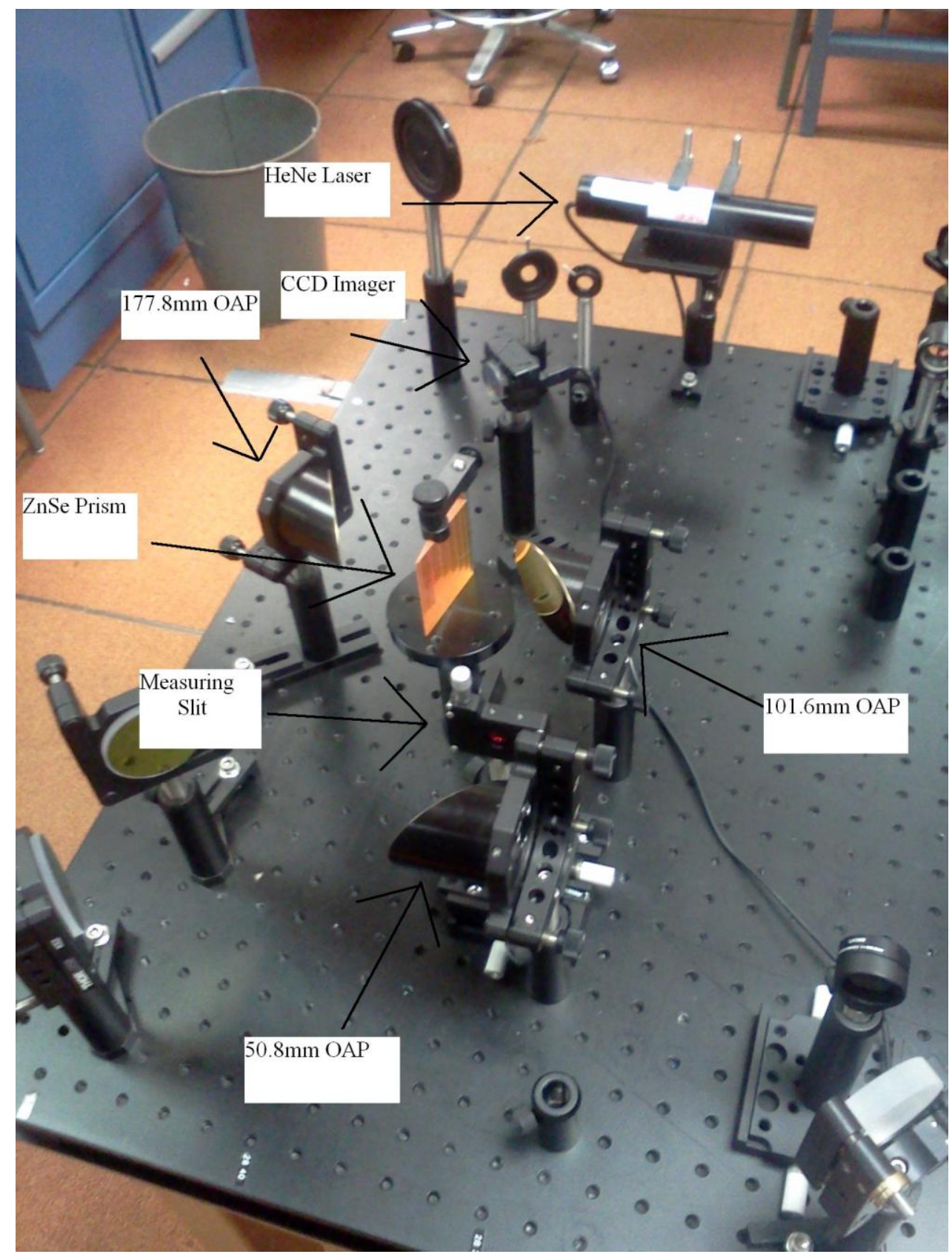

Figure 4: Final layout of aligned optical components. The ZnSe prism and gold-coated OAP mirrors are all visible. 


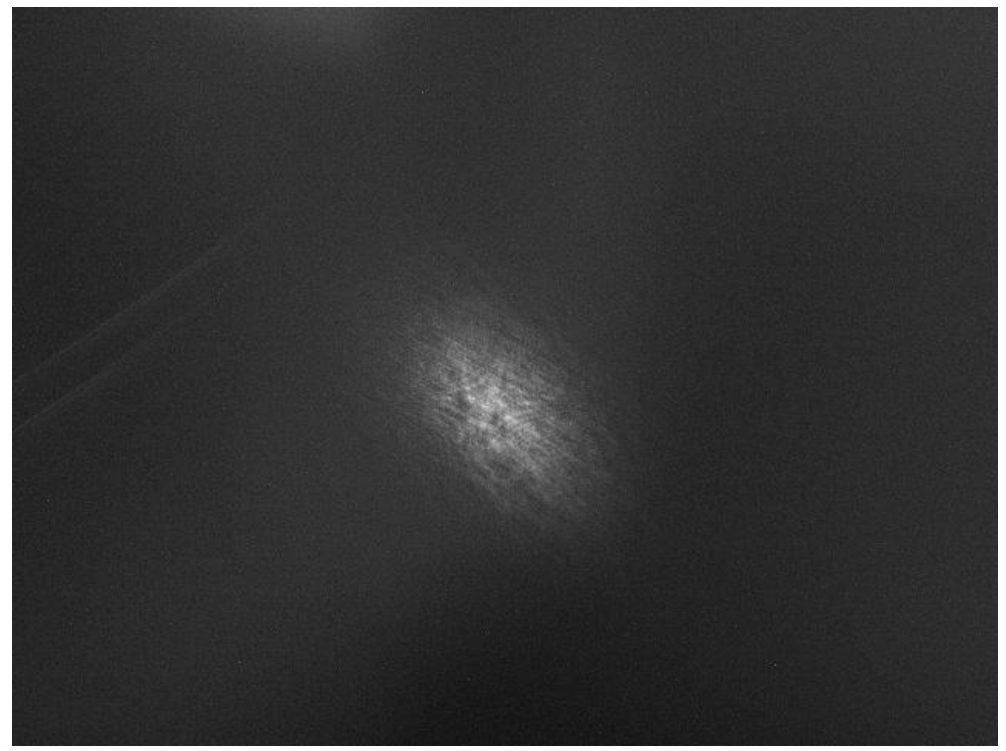

Figure 5: CCD camera image of the beam under 50 ODs of attenuation taken at the focal point of the third OAP mirror. Note its approximately Gaussian composition and the absence of severe aberrational effects. 
Table 1: Focal Lengths of Relevant Optical Equipment

\begin{tabular}{|c|c|}
\hline \multicolumn{2}{|c|}{ Focal Lengths } \\
\hline Optical Equipment & $\begin{array}{c}\text { Focal Length } \\
\text { mm }\end{array}$ \\
\hline Plano-Concave Lens & -30 \\
\hline Plano-Convex Lens & 500 \\
\hline OAP 1 & 50.8 \\
\hline OAP 2 & 151.6 \\
\hline OAP 3 & 177.8 \\
\hline
\end{tabular}

